\title{
The Potential Role of Elk-3/Egr-1 Signaling Pathway in the Epithelial- Mesenchymal Transition during Liver Fibrosis
}

\author{
In Hee Kim
}

Department of Internal Medicine, Research Institute of Clinical Medicine, Chonbuk National University Hospital, Chonbuk National University Medical School, Jeonju, Korea

See "Elk-3 Contributes to the Progression of Liver Fibrosis by Regulating the Epithelial-Mesenchymal Transition" by Tian Zhu Li, et al. on page 102, Vol. 11. No. 1, 2017

Liver fibrosis is characterized by a dysregulated wound healing process that results in excessive deposition of the extracellular matrix (ECM), mainly collagen type I, and scar formation. Hepatic myofibroblasts are part of the heterogenous cell group, which has a fundamental role for the development of liver fibrosis. ${ }^{1}$ However, the origin of myofibroblasts in the fibrotic liver has not been fully elucidated and is still under heated debate, despite extensive research. ${ }^{2}$ Although activated hepatic stellate cells have been considered as the major source, since they are the main ECM-producing cells in the injured liver, previous studies suggested that the epithelial-mesenchymal transition (EMT) is one of the mechanisms that give rise to hepatic myofibroblasts in liver fibrosis. ${ }^{3}$

During EMT, epithelial cells lose key epithelial features, such as apical-basal polarity, intercellular adhesion complexes, and adherence to a basal basement membrane, while gradually obtaining multiple mesenchymal phenotypes, including spindleshaped appearance, increased cell motility, invasiveness, and increased production of ECM components. ${ }^{3}$ EMT is divided into three distinct categories: type 1 occurring in development, type 2 in fibrosis, and type 3 in cancer and metastasis. ${ }^{3}$ Type 2 EMT has been associated with organ fibrosis and regeneration, occurring in the liver, lung, kidney and intestine. The cells that undergo EMT show a loss of epithelial adhesion protein, Ecadherin, counterbalanced by the aberrant expression of $\mathrm{N}$ cadherin. Fibroblast-specific protein 1 (FSP1; also known as S100A4), $\alpha$-smooth muscle actin ( $\alpha$-SMA), and collagen 1 are characterized markers of the mesenchymal products generated by the EMT during organ fibrosis development. ${ }^{3}$ Other biomark- ers, including vimentin, desmin, fibronectin, and discoidin domain receptor 2 have been used to demonstrate the epithelial cells undergoing EMT. ${ }^{4}$ Through the EMT process, epithelial cells eventually lose their epithelial markers (E-cadherin and zonula occludens-1), acquiring a fully fibroblastic mesenchymal phenotype ( $\mathrm{N}$-cadherin, vimentin, FSP1, collagen-1, and $\alpha$-SMA).

Transforming growth factor $\beta$ (TGF- $\beta$ ) is believed to be a potent inducer of EMT and a well-established profibrogenic cytokine in liver fibrosis. ${ }^{5}$ Hepatocyte EMT was observed in the primary hepatocytes incubated with TGF- $\beta 1$ and primary hepatocytes from $\mathrm{CCl}_{4}$-induced cirrhotic liver. ${ }^{6,7}$ In the TGF $\beta$ signaling pathway, active TGF- $\beta 1$ ligands initiate the signaling process by binding to TGF- $\beta$ receptor type I (T $\beta R I)$ and T $\beta R I I$ serine/threonine kinases. ${ }^{3}$ T $\beta$ RI phosphorylates Smad2 and Smad3, which form a complex with Smad4 and translocate to the nucleus. Smad proteins convey signals from TGF- $\beta$ to the nucleus. Once in the nucleus, the complex of Smads can regulate the transcription of target genes. The activation of several Smad independent pathways have been identified to be a crucial component for EMT induction by TGF- $\beta$, including phosphoinositide 3-kinase (PI3K)-Akt, focal adhesion kinase, p38 the mitogen activated protein kinases (MAPK), and ERK. In addition, recent studies have implicated Krüppel-like factor-8, hyaluronan synthase 2, and microRNA miR-203 as critical regulators for EMT. ${ }^{3}$

Li et al. ${ }^{8}$ investigated novel molecular mechanisms involved in the EMT of hepatocytes and liver fibrosis. They demonstrated that the expression levels of Elk-3 and early growth response-1 (Egr-1) were significantly increased during the TGF- $\beta 1$-induced

Correspondence to: In Hee Kim

Department of Internal Medicine, Research Institute of Clinical Medicine, Chonbuk National University Hospital, Chonbuk National University Medical School, 20 Geonji-ro, Deokjin-gu, Jeonju 54907, Korea

Tel: +82-63-250-1677, Fax: +82-63-254-1609, E-mail: ihkimmd@jbnu.ac.kr pISSN 1976-2283 eISSN 2005-1212 https://doi.org/10.5009/gnl16564

@ This is an Open Access article distributed under the terms of the Creative Commons Attribution Non-Commercial License (http://creativecommons.org/licenses/by-nc/4.0) which permits unrestricted non-commercial use, distribution, and reproduction in any medium, provided the original work is properly cited. 
EMT of hepatocytes, in both the $\mathrm{CCl}_{4}$-induced mouse liver fibrotic tissues and in the human liver cirrhotic tissues. E26 transformation-specific (ETS) proteins form one of the largest families of signal-dependent transcriptional regulators, which mediate cellular proliferation, differentiation, and tumorigenesis. ${ }^{9}$ Elk-3 (Net/Sap-2/Erp) is a member of the ternary complex factors subfamily of ETS proteins, along with Elk-1 and Sap-1. Elk-3 plays an important role in wound healing, angiogenesis, cell migration, and tumorigenesis. Elk-3 is activated by the expression of Ras and phosphorylated by ERK and p38. Egr1 , a zinc finger-containing transcription factor, is immediately expressed in response to a variety of stimuli, such as growth factors and lipopolysaccharide. It has been reported that Egr1 can regulate genes involved in the wound-healing process and immune response. However, to the best of our knowledge, there have not been any studies investigating the role of Elk3 and Egr-1 during EMT of hepatocyte in liver fibrosis. Li et al. ${ }^{8}$ demonstrated that the expression levels of Elk-3 and Egr-1 were significantly increased during TGF- $\beta 1$-induced EMT of hepatocytes, in both the $\mathrm{CCl}_{4}$-induced mouse liver fibrotic tissues and in the human liver cirrhotic tissues. Furthermore, they investigated the molecular relationships among Elk-3, Egr-1, and the p38 MAPK pathway during EMT in hepatocytes. According to the results, the silencing of Elk-3 and inhibition of the RasElk-3 pathway with an inhibitor suppressed the expression of EMT-related markers. Moreover, Elk-3 expression was regulated by p38 MAPK phosphorylation during EMT. Therefore, the study by Li et al. ${ }^{8}$ suggested Elk-3/Egr-1 MAPK signaling as a novel molecular pathway in the progression of liver fibrosis via the regulation of hepatocyte EMT.

However, since recent studies using "lineage tracing" method reported evidence against EMT in the liver, the EMT theory is one of the most controversial issues in this field of research. ${ }^{10}$ Taura et al. ${ }^{11}$ bred triple transgenic mice expressing ROSA26 stop $\beta$-galactosidase ( $\beta$-gal), Albumin Cre, and collagen $\alpha$ (I) green fluorescent protein (GFP), in which the hepatocytederived cells are permanently labeled by $\beta$-gal and type I collagen-expressing cells are labeled by GFP. The study examined the expression of four different mesenchymal markers (FSP 1, $\alpha$-SMA, vimentin, and desmin) in the primary hepatocyte from the untreated and $\mathrm{CCl}_{4}$-treated livers and fibrotic liver tissues induced by $\mathrm{CCl}_{4}$ injections. According to the results, type I collagen-producing cells do not originate from the hepatocytes. Hepatocytes in vivo neither acquire the mesenchymal marker expression nor exhibit a morphological change that clearly distinguishes from the normal hepatocytes. Thus, these findings strongly challenge the concept that hepatocytes in vivo acquire a mesenchymal phenotype through EMT to produce ECM in liver fibrosis. However, these contradictory studies against EMT in liver fibrosis also has some methodological concerns, includ- ing incomplete efficiency of Cre-mediated recombination, use of only a few mesenchymal markers on immunostaining, and discordance of experimental models from human chronic liver disease. These criticisms cannot completely rule out the possible role of EMT in liver fibrosis. Indeed, many studies have still been investigating the possibility of EMT and related molecular mechanisms in hepatic fibrogenesis. Therefore, regardless of the discourse on EMT, it is necessary for future research on EMT to provide noble insights into the plasticity of cellular phenotypes, molecular signaling pathways, and any possible therapeutic targets in liver fibrosis.

\section{CONFLICTS OF INTEREST}

No potential conflict of interest relevant to this article was reported.

\section{REFERENCES}

1. Novo E, di Bonzo LV, Cannito S, Colombatto S, Parola M. Hepatic myofibroblasts: a heterogeneous population of multifunctional cells in liver fibrogenesis. Int J Biochem Cell Biol 2009;41:20892093.

2. Lua I, Asahina K. The role of mesothelial cells in liver development, injury, and regeneration. Gut Liver 2016;10:166-176.

3. Zhao YL, Zhu RT, Sun YL. Epithelial-mesenchymal transition in liver fibrosis. Biomed Rep 2016;4:269-274.

4. Zeisberg M, Neilson EG. Biomarkers for epithelial-mesenchymal transitions. J Clin Invest 2009;119:1429-1437.

5. Bi WR, Yang CQ, Shi Q. Transforming growth factor-beta1 induced epithelial-mesenchymal transition in hepatic fibrosis. Hepatogastroenterology 2012;59:1960-1963.

6. Nitta T, Kim JS, Mohuczy D, Behrns KE. Murine cirrhosis induces hepatocyte epithelial mesenchymal transition and alterations in survival signaling pathways. Hepatology 2008;48:909-919.

7. Kaimori A, Potter J, Kaimori JY, Wang C, Mezey E, Koteish A. Transforming growth factor-beta1 induces an epithelial-to-mesenchymal transition state in mouse hepatocytes in vitro. J Biol Chem 2007;282:22089-22101.

8. Li TZ, Kim SM, Hur W, et al. Elk-3 contributes to the progression of liver fibrosis by regulating the epithelial-mesenchymal transition. Gut Liver 2017;11:102-111.

9. Mavrothalassitis G, Ghysdael J. Proteins of the ETS family with transcriptional repressor activity. Oncogene 2000;19:6524-6532.

10. Taura K, Iwaisako K, Hatano E, Uemoto S. Controversies over the epithelial-to-mesenchymal transition in liver fibrosis. J Clin Med 2016;5:9.

11. Taura K, Miura K, Iwaisako K, et al. Hepatocytes do not undergo epithelial-mesenchymal transition in liver fibrosis in mice. Hepatology 2010;51:1027-1036. 